\title{
Buccal oxycodone reduced pain more than placebo in children with acute undifferentiated abdominal pain
}

Kokki H, Lintula H, Vanamo K, et al. Oxycodone vs placebo in children with undifferentiated abdominal pain: a randomized, doubleblind clinical trial of the effect of analgesia on diagnostic accuracy. Arch Pediatr Adolesc Med 2005;159:320-5.

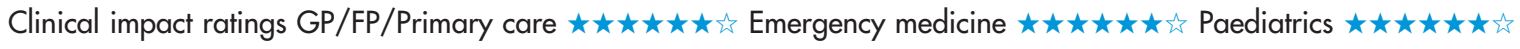

In children with acute undifferentiated abdominal pain, is buccal oxycodone more effective than placebo for reducing pain without altering clinical signs or outcome?

\section{METHODS}

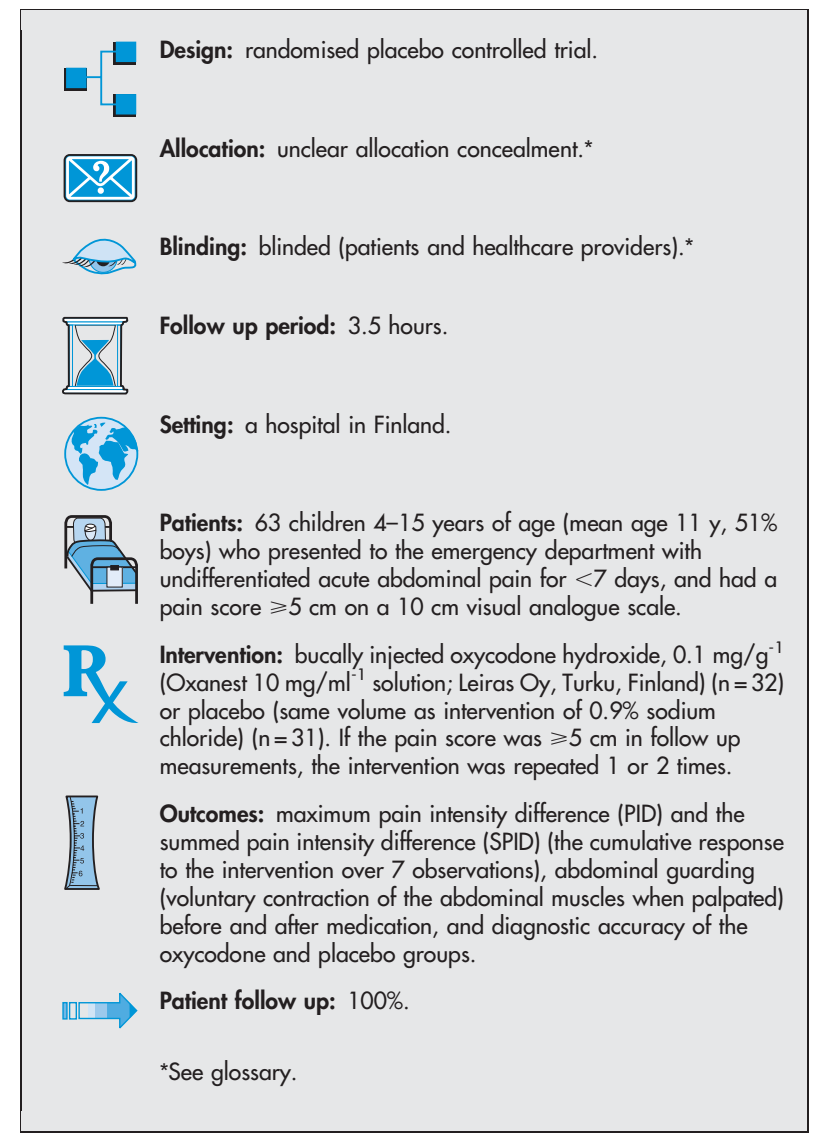

\section{MAIN RESULTS}

Pain was reduced in both the oxycodone and placebo groups. The SPID was higher in children who received oxycodone than in those

For correspondence: Dr H Kokki, Kuopio University Hospital, Kuopio, Finland. hannu.kokki@kuh.fi

Source of funding: not stated. who received placebo (table). Predose abdominal guarding occurred in 16 children in the oxycodone group (50\%) and 13 children in the placebo group (42\%). Of the 7 children that altered guarding, 6 were in the oxycodone group with 3 becoming positive. Guarding disappeared in more children in the oxycodone group than the placebo group ( $3 v 1, \mathrm{p}=0.05)$. Diagnostic accuracy after treatment increased from baseline in the oxycodone group (72\% to $88 \%)$ and remained at $84 \%$ in the placebo group.

\section{CONCLUSIONS}

In children with acute undifferentiated abdominal pain, buccal oxycodone reduced pain more than placebo. Oxycodone did not interfere with clinical signs or surgical diagnoses.

\section{Commentary}

T he study by Kokki et al attempts to fill an important void in the literature about treating children with acute undifferentiated abdominal pain. Physicians who initially evaluate children with acute abdominal pain struggle with the issue of preserving clinical findings (and hence the diagnostic accuracy for the surgical consultant) while providing pain relief for a suffering child. The lack of an evidencebased approach can occasionally create friction between paediatric emergency physicians and paediatric surgeons. Efforts by the authors to address this clinical dilemma are applauded.

The current prospective, double blind, placebo controlled trial, which had clear inclusion and exclusion criteria, was planned to detect a 2 fold difference in SPID between treatment and placebo groups. Although this is a sound study design based on an important outcome, preserving diagnostic accuracy is more critical. Given the small difference in the diagnostic accuracy between groups $(4 \%)$, a much larger sample size would be required to show convincingly that patient safety is not compromised.

The advent of increased accuracy of imaging prompts a shift in the current practice of diagnosing acute appendicitis to a radiographic diagnosis. Thus, the downside of using analgesics in this population is lessened. Given the above argument, pain control should not be withheld from a child with clear signs and symptoms for or against appendicitis or when imaging is planned. A good rapport and trust between paediatric emergency physicians and their surgical colleagues would enhance this clinical approach.

Magdy W Attia, MD Alfred I duPont Hospital for Children Wilmington, Delaware, USA

Oxycodone $v$ placebo for acute undifferentiated abdominal pain in children*

\begin{tabular}{llll}
\hline Outcomes at 3.5 hours & Oxycodone & Placebo & Mean difference $(95 \% \mathrm{Cl})$ \\
\hline Mean SPID $(\mathrm{cm})$ & 22 & 9 & $13(2$ to 24$)$ \\
Mean maximal PID $(\mathrm{cm})$ & 3.7 & 2.7 & $1.0(-0.4$ to 2.4$)$ \\
\hline
\end{tabular}

*SPID = summed pain intensity difference (cumulative response over 7 observations); PID = pain intensity difference (peak of the analgesic effect); other abbreviations defined in glossary. 